\title{
ASSOCIAÇÃO ENTRE TAMANHO E POTENCIAL PROLIFERATIVO EM NEURINOMAS DO ACÚSTICO
}

\author{
Oswaldo Inácio de Tella1, João Norberto Stavale², Marco Antonio Herculano³, \\ Manoel Antonio de Paiva Neto ${ }^{4}$, Franz Jooji Onishis, \\ Francisco de Assis Vaz Guimarães Filho5, Luciano Ricardo França e Silva ${ }^{5}$
}

\begin{abstract}
RESUMO - Schwanomas do acústico são os tumores mais freqüentes localizados no ângulo pontocerebelar. Os mecanismos moleculares que levam a sua geração e crescimento ainda não são bem conhecidos. Várias características clínicas, radiológicas e imuno-histoquímicas já foram estudadas e correlacionadas ao c rescimento tumoral. Estudamos e correlacionamos aspectos clínicos e imuno-histoquímicos (MIB-1) de 11 schwanomas do acústico operados no Hospital São Paulo/UNIFESP. O tamanho dos tumorescorrelacionouse com o índice proliferativo (Ki-67), não havendo correlação com significância estatística entre a idade dos pacientes, duração dos sintomas e índice proliferativo.
\end{abstract}

PALAVRAS-CHAVE: Ki-67, índice proliferativo, neurinoma do acústico, MIB-1.

\begin{abstract}
Size and proliferative index correlation in acoustic neuromas
ABSTRACT - Acoustic neuromas are the most common tumors in the cerebellopontine angle. The molecular mechanisms involved in generation and growth of these tumors are not completly elucidated. Many radiological, clinic and imunohistochemystry data were correlated to tumor growth. We studied 11 acoustic n e u romas surgically treated at Hospital São Paulo/UNIFESP and correlated clinical and radiological data with proliferative index (Ki-67). The size of the tumors were positively correlationated with proliferative index. No other correlation had statistic significativity.
\end{abstract}

KEY WORDS: Ki-67, proliferative index, acoustic neuroma, MIB-1.

Neurinomas do acústico são os tumores mais freqüentes do ângulo pontocerebelar. Cerca de 2000 a 3000 casos novos deste tumor são diagnosticados anualmente nos EUA ${ }^{1}$. Geralmente tem crescimento lento, em média 1 a $5 \mathrm{~mm}$ por ano, e se originam na bainha do nervo vestibular perto da junção mielinoglial, próximo ao poro acústico interno. São tumores bem circunscritos e encapsulados por epineuro ${ }^{2-4}$. Atualmente, a ressonância magnética (RM) e tomografia computadorizada (TC) são inadequadas para prever crescimento tumoral a não ser quando feitas de maneira seriada. O melhor entendimento das características de crescimento destes tumores é importante para um melhor entendimento da biologia tumoral e poder ajudar na formulação de decisões clínicas como sobre o tratamento ou observação; ou ainda a freqüência a serem feitos os exames de imagem.

Testes imuno-histoquímicos trouxeram novas possibilidades na estimativa de crescimento destes tumores, pois permitem a determinação de várias fases do ciclo celular, refletindo a capacidade proliferativa destes tumores. O Ki-67, antígeno nuclear presente somente na divisão nuclear, é o que aparece nas fases G1 (intervalo - "gap" - que se segue a mitose, após a replicação do DNA), G2 (intervalo que $p$ recede a mitose) e $M$ (intervalo do ciclo celular de eucariotos, no qual ocorre a divisão do núcleo e do citoplasma) do ciclo celular, exceto na fase de repouso G0 (estado de recessão do ciclo de divisão celular de eucariotos) ${ }^{5}$.

O presente estudo visa correlacionar características

${ }^{1} \mathrm{P}$ rofessor Adjunto Livre Docente da Disciplina de Neurocirurgia da Universidade Federal de São Paulo (UNIFESP) e Neurocirurgião do Hospital Professor Edmundo Vasconcelos, São Paulo SP, Brasil; ${ }^{2}$ P rofessor Adjunto da Disciplina de Anatomia Patológica da UNIFESP e Patologista do Hospital Professor Edmundo Vasconcelos; ${ }^{3}$ P rofessor Adjunto da Disciplina de Neurocirurgia da Faculdade de Medicina de Jundiaí e Neurociru rgião do Hospital Professor Edmundo Vasconcelos; ${ }^{4}$ Médico Neurociru rgião do Hospital São Paulo/UNIFESP e Neuro c i ru rgião do Hospital Professor Edmundo Vasconcelos; ${ }^{5}$ Médico Residente da Disciplina de Neuro ciru rgia da UNIFESP.

Recebido 18 Março 2005, recebido na forma final 25 Agosto 2005. Aceito 13 Outubro 2005.

Dr. Osvaldo Inácio de Tella Jr. - Rua São Paulo Antigo 145 / bloco F / Apto 11 - 05864-010 São Paulo SP - Brasil. E-mail: detella@ globo.com.br 
clínicas e radiológicas selecionadas de schwanomas com os resultados do índice proliferativo.

\section{MÉTODO}

Este estudo inclui 11 pacientes consecutivos, operados na UNIFESP-EPM, no período de janeiro de 2002 a dezembro de 2003 e com diagnóstico anatomopatológico de schwanoma do nervo vestibular unilateral. Foram excluídos pacientes port a $\mathrm{d}$ o res de ne uro $\mathrm{f} i \mathrm{~b}$ romatose. Foi considerada a duração dos sintomas e idade dos pacientes no momento do diagnóstico. Todos os pacientes foram submetidos a estudo de RM de crânio onde o maior diâmetro tumoral de cada tumor foi medido (incluindo porção intracanalicular). Em todos os casos estudados utilizou-se técnica de imunohistoquímica para determinação da expressão do antígeno Ki-67.
Os testes foram conduzidos e $m$ tecidos estocados em parafina. A imuno-histoquímica foi realizada segundo método descrito por Cattoretti et al. ${ }^{6}$, utilizando-se as especificações contidas no Kit de peroxidase universal (Immunotech, Marselha, França). Os fragmentos de tumor foram desparafinizados e hidratados com banhos de xileno e etanol. Seguiu-se a utilização de citrato tamponado. Após isto, foram processados em microondas por 25 minutos. As lâminas foram então incubados com soro normal seguido do anticorpo primário MIB-1 (Immunotech). O cromógeno utilizado foi diaminobenzidina e acetilcarbazolamina. As secções foram então coradas com hematoxilina. Para cada série de reações, um controle negativo foi realizado omitindo o MIB-1 e metanol. A célula foi considerada positiva quando parte ou todo o núcleo foi corado. O número de células positivas e negativas foi contado em um total de 500 células por campo em seis campos (seis campos de 500). O índice

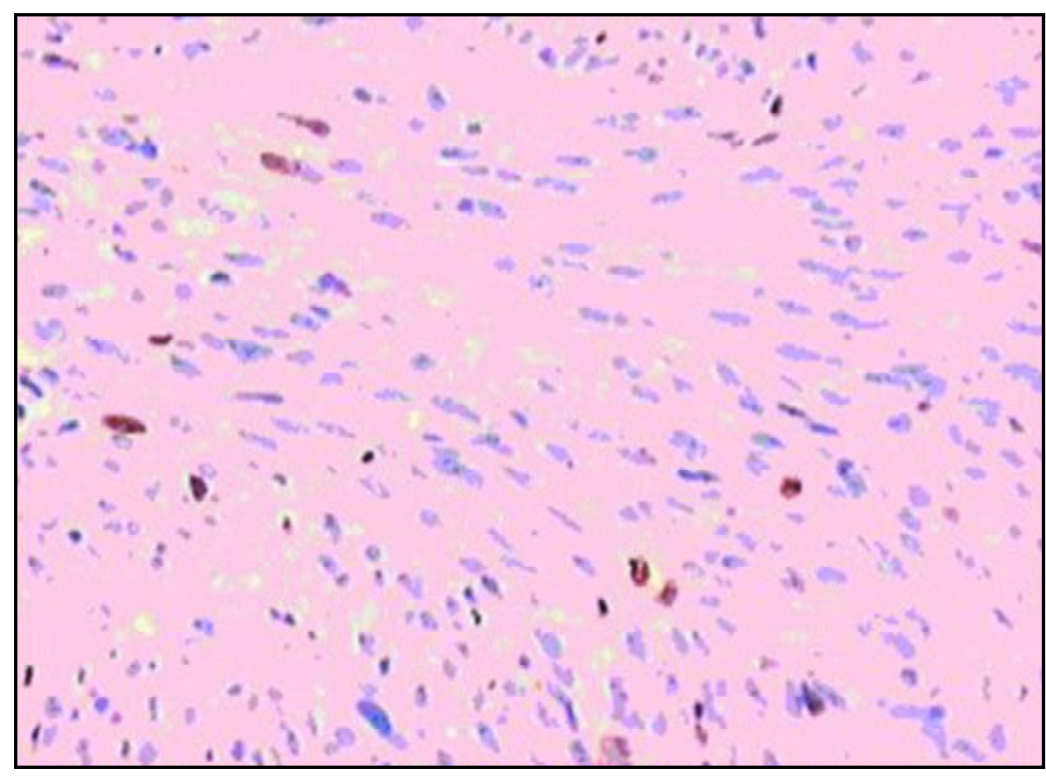

Fig 1. Imuno-histoquímica para Ki-67 usando anticorpo Ki-67 (1:30, DAKO) 100x.

Fig 2. Imuno-histoquímica para Ki-67 usando anticorpo Ki-67 (1:30, DAKO) 400x.

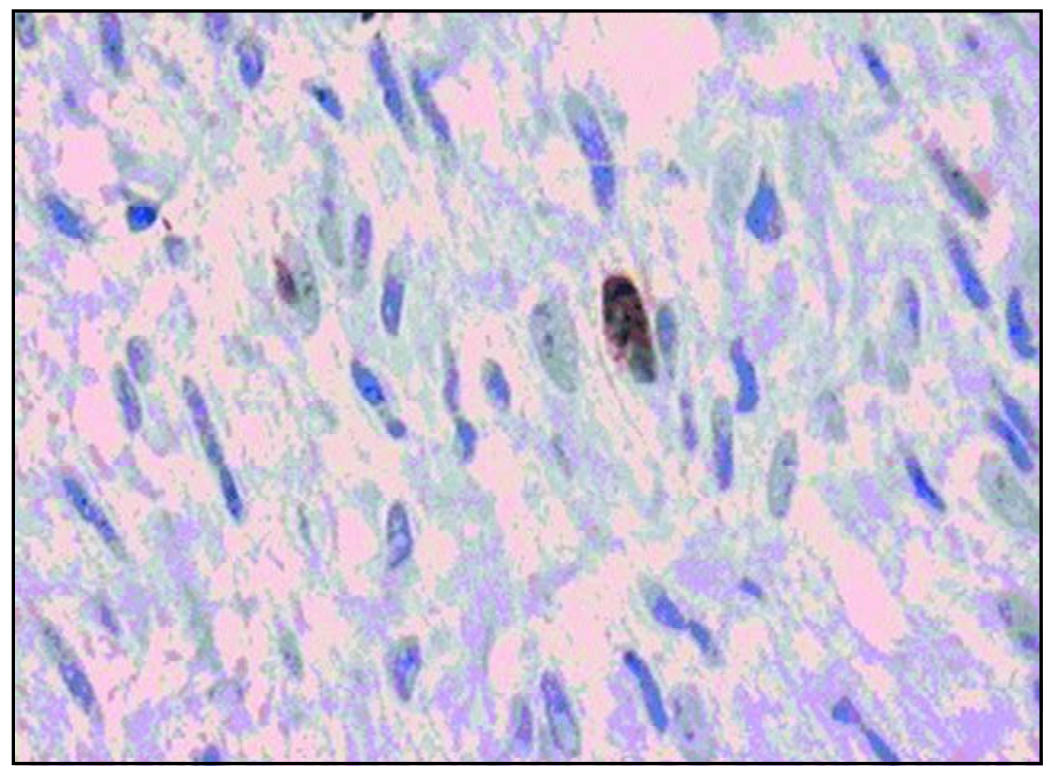






Fig 3. Gráfico mostrando os dados analisados estatisticamente pelo teste de Spearman, considerando como estatisticamente significativo os valores de $p<0,05$.

proliferativo foi definido como a proporção de células positivas em relação ao total avaliado (3000) (Figs 1 e 2).

Os dados obtidos foram analisados estatisticamente utilizando-se testes não paramétricos (Spearman), considerando como estatisticamente significativo os valores de $\mathrm{p}<0,05$ (Fig 3).

O material foi submetido a imuno-histoquímica no Serviço de Patologia do Hospital Professor Edmundo Vascon- celos, tendo para tanto a aprovação do Conselho de Ética em Pesquisa dessa Instituição.

\section{RESULTADOS}

A tabela mostra os dados clínicos e índice proliferativo dos pacientes. Todos os pacientes pertenciam ao sexo feminino, tendo sido operados por via suboccipital ret romastoidéia. O diagnóstico de schwanoma foi confirmado em todos os casos através do tecido fixado em formalina e coloração HE. O índice proliferativo foi analisado em 11 amostras. O Ki-67 variou de 0 a $1 \%$, média 0,35. As complicações cirúrgicas principais foram paresia facial periférica que variou de House e Brackman Grau II em um caso, Grau III em dois casos, Grau V em cinco e Grau VI três casos (Quadro). A audição não foi pre s e rvada em nenhum caso.

Seis tumores localizavam-se a direita e cinco á esquerda.A idade variou de 36-79 anos (média 55,3 anos). Houve correlação negativa do índice prolifera tivo com a idade dos pacientes $(r=-0,206)$, porém sem significância.

O diâmetro tumoral máximo (incluindo porção intracanalicular) variou de 23 a $40 \mathrm{~mm}$ com média de 32,2 mm. Observou-se correlação positiva entre tamanho tumoral e índice proliferativo $(r=0,535)$ com significância estatística $(p<0,05)$.

A duração dos sintomas antes do diagnóstico ser estabelecido variou de um a 13 meses, com média de 7,1 meses. Os sintomas principais foram hipoacusia em seis casos, vertigem em quatro casos e cefaléia

Tabela. Distribuição dos casos clínicos, segundo sexo, idade, duração dos sintomas em meses, tamanho do tumor em centímetros e o índice do Ki-67.

\begin{tabular}{ccccc}
\hline Caso & Sexo/ldade & $\begin{array}{c}\text { Duração dos } \\
\text { sintomas (meses) }\end{array}$ & $\begin{array}{c}\text { Tamanho } \\
\text { tumor }(\mathrm{cm})\end{array}$ & Ki-67 \\
\hline 1 & $\mathrm{~F} / 79$ & 6 & 3,8 & 0,3 \\
2 & $\mathrm{~F} / 55$ & 1 & 3,7 & 0,5 \\
3 & $\mathrm{~F} / 50$ & 9 & 3,9 & 0 \\
4 & $\mathrm{~F} / 55$ & 6 & 3,2 & 1 \\
5 & $\mathrm{~F} / 66$ & 9 & 2,4 & 0 \\
6 & $\mathrm{~F} / 36$ & 12 & 2,3 & 0 \\
7 & $F / 53$ & 1 & 4 & 1 \\
8 & $F / 71$ & 15 & 3,0 & 0 \\
9 & $F / 59$ & 3 & 2,4 & 0 \\
10 & $F / 45$ & 4 & 3 & 0,5 \\
11 & $F / 40$ & 3 & 3,8 & 0,6 \\
\hline
\end{tabular}


Quadro. Escala de House e Brackman.

\begin{tabular}{ll}
\hline Grau & Quadro clínico \\
\hline I & Função normal em todos territórios \\
II & $\begin{array}{l}\text { Disfunção leve. Ligeira ou leve debilidade da muscula- } \\
\text { tura, detectada na inspeção meticulosa. Em repouso } \\
\text { simetria normal. Sem sincinesias, nem contraturas ou } \\
\text { espasmos faciais }\end{array}$ \\
III & $\begin{array}{l}\text { Disfunção moderada. Diferença clara entre ambos la- } \\
\text { dos sem ser desfigurante. Incompetência para o fe- } \\
\text { chamento palpebral completo; existe movimento de } \\
\text { região frontal, assimetria da comissura labial em mo- } \\
\text { vimentos máximos. Em repouso simetria e tonus } \\
\text { normal } \\
\text { IV } \quad \begin{array}{l}\text { Disfunção moderadamente severa. Debilidade e/ou } \\
\text { assimetria desfigurante. Em repouso simetria e tonus } \\
\text { normal. Não existe movimento de região frontal; im- } \\
\text { possibilidade para fechar o olho totalmente. Sincine- } \\
\text { sias. Espasmo facial. }\end{array} \\
\text { Disfunção severa. Somente ligeira atividade motora } \\
\text { perceptível. Em repouso assimetria. }\end{array}$ \\
$\begin{array}{l}\text { Paralisia total. Não existe movimento facial. Perda } \\
\text { total do tonus. }\end{array}$ \\
\hline
\end{tabular}

em um caso. Todos os pacientes, exceto o Caso 8, com duração dos sintomas maior que seis meses tiveram $\mathrm{Ki}-67=06$. Os cinco pacientes que apresentaram sintomas com duração menor ou igual a seis meses apresentaram Ki-67 que variou de 0,3 a 1. Correlacionando estes dados observou-se relação negativa entre a duração dos sintomas e $o$ índice proliferativo ( $r=$ $-0,487$ / $p>0,05)$.

\section{DISCUSSÃO}

As características anatomopatológicas dos schwanomas do acústico são idênticas de todos outros schwanomas. Em casos raros, o exame histopatológico pode revelar achados não típicos de schwanomas benignos como: lesões atípicas ou malignas com índices mitóticos altos, figuras de mitose atípicas e pobre diferenciação celular ${ }^{7}$. O mecanismo molecular que gera sua formação e crescimento ainda é desconhecido.

A incidência de neurinomas do acústico assintomáticos tem freqüência de $0,57 \%$ a $0,87 \%$, segundo estudo de ossos temporais em necropsia. Isto leva a crer que menos de $1 \%$ de todos os neuromas do acústico têm crescimento suficiente para se tornar clinicamente ativos. Estudos mostrando RM seriada sugerem que em $40 \%$ casos não há crescimento tumoral ou pode haver até diminuição de seu tamanho. Crescimento maior que $1 \mathrm{~cm} /$ ano pode ser observado em até $13 \%$ dos tumores. Com o advento de técnicas de imagem mais sofisticadas, a chance de se diagnosticar um tumor que não será sintomático tem crescido significativamente.

Vários estudos já foram realizados para se avaliar o crescimento destes tumores. Em metanálise realizada com 571 pacientes com média de idade de 64 anos e seguimento médio de três anos, mostrou crescimento em $54 \%$ dos casos $^{8}$. Charabi et al., relataram crescimento médio de $2,4 \mathrm{~mm} / \mathrm{ano}$ em 108 casos estudados, $34 \%$ necessitando tratamento durante 0 período ${ }^{9}$. Rosenberg ${ }^{1}$, em 80 pacientes, observou crescimento em $57,8 \%$ dos casos em seguimento de 4,4 anos, somente $5,7 \%$ dos pacientes maiores que 65 anos necessitaram de tratamento cirúrgico durante o acompanhamento.

Em 1950, Olivecrona reviu seus resultados em ressecção parcial de neuromas do acústico e concluiu que alguns tumores param de crescer após ressecção subtotal. No entanto, alguns anos após, ele relatou a recorrência em $21 \%$ dos casos; também observou menor mortalidade em longo prazo quando os tumores eram ressecados totalmente ${ }^{10}$. House relatou recorrência de $23 \%$ em 56 ressecções subtotais, um terço destes precisando ser reoperados em quatro anos ${ }^{11}$. O índice de re co rrência variou de 12,5 a $45 \%$ em vários estudos. Rosenberg demonstrou que $68 \%$ dos pacientes não apresentaram crescimento tumoral após ressecção subtotal. $O$ índice de recorrência em tumores com ressecção total varia de 0,4-2\% $\%^{1,3,10-12}$.

A proliferação celular não é o único fator envolvido no crescimento dos schwanomas vestibulares; estes também podem se expandir devido a degeneração cística e sangramento. Porém, é o mais fácil de ser avaliado ${ }^{5,13}$. A desvantagem é que só pode ser utilizado após tratamento cirúrgico. O Ki-67 tem sido usado exaustivamente como marcador de proliferação celular expressando a proliferação ou índice de crescimento de vários tumores. Alguns autores mostraram uma leve tendência do índice proliferativo diminuir com a idade do paciente ${ }^{14}$. Charabi et al. ${ }^{15,16}$ acharam relação inversa significativa entre a duração dos sintomas e índice proliferativo em duas series de 21 e 124 tumores, mas nenhuma correlação entre tamanho do tumor e proliferação. O nosso material não mostrou correlação estatisticamente significativa entre duração dos sintomas e índice proliferativo.

Pela presente casuística houve correlação positiva entre o tamanho tumoral e índice proliferativo $(p<$ 
$0,05)$, não se observando correlação estatisticamente significativa entre índice proliferativo e idade dos pacientes, provavelmente por ser a nossa série. Bedavanija et al. ${ }^{17}$ mostraram que neurinomas grandes exibem índices proliferativos e de crescimento maiores que tumores pequenos, indicando como tamanho de corte $18 \mathrm{~mm}$. Sugeriram que tumores grandes parecem crescer mais rapidamente que tumores pequenos. Também demonstraram que tumores pequenos e com baixo índice proliferativo ocorrem em todas as idades, com tendência a uma atividade proliferativa menor nos pacientes acima de 50 anos. Su$g$ e rem que tumores agressivos são raros em idosos, enquanto tumores de crescimento lento podem aparecer em qualquer idade. A correlação positiva entre tamanho tumoral e velocidade de crescimento foi confirmada em outros estudos ${ }^{1,3,5}$. Aguiar et al. também observaram índices mitóticos maiores em tumores císticos e pacientes mais jovens ${ }^{5}$. Estes autores sugerem tratamento ativo no subgrupo de pacientes menores que 50 anos com tumores grandes. Fucci et al. mostraram índice proliferativo maior em schwanomas atípicos que têm tendência maior de recorrência sendo necessário um acompanhamento mais próximo ${ }^{18}$.

A aferição do índice proliferativo ainda pode ser útil quando há ressecção parcial do tumor, visandose presenação de nervos cranianos. Permite a seleção de pacientes que devem ser acompanhados com investigação radiológica mais freqüente, facilitando assim a detecção de recorrência ${ }^{14,19,20}$.

Em conclusão, o anticorpo Ki-67 tem sido útil em demonstrar a atividade proliferativa dos schwanomas do acústico. Características clínico-radiológicas como duração dos sintomas, idade dos pacientes e tamanho tumoral podem estar correlacionadas com o crescimento destes tumores, sendo demonstrada correlação positiva estatisticamente significativa somente quando foi comparado o tamanho do tumor e índice proliferativo nos casos estudados neste trabalho.

\section{REFERÊNCIAS}

1. Rosenberg SI. Natural history of acoustic neuromas. Laryngoscope 2000;110:497-508.

2. Wazen J, Silverstein H, Norrell H, Besse B. Preoperative and postoperative growth rates in acoustic neuromas documented with CT scanning. Otolaryngol Head Neck Surg 1985;93:151-155.

3. Silverstein H, McDaniel A, Norrell H, Wazen J. Conservative management of acoustic neuroma in the elderly patient. Laryngoscope 1985;95: 766-770.

4. Zöllner C, Bockenheimer S. The growth rate of acoustic neuromas: a report of three cases. Arch Otorhinolaryngol 1985;241:259-264.

5. Aguiar PH, Tatagiba M, Dankoweit-Timpe E, Matthies C, Samii M, Ostertag H. Proliferative activity of acoustic neurilemomas without neurofibromatosis determined by monoclonal antibody MIB 1. Acta Neurochir (Wien) 1995;134:35-39.

6. Cattoretti G, Becker MH, Key G, et al. Monoclonal antibodies against recombinant parts of the Ki-67 antigen (MIB 1 and MIB 3) detect p roliferating cells in microwave-processed formalin-fixed paraff in sections. J Pathol 1992;168:357-363.

7. Light JP, Roland JT, Fishman A, Miller DC, Cohen NL. Atypical and low-grade malignant vestibular schwannomas: clinical implications of proliferative activity. Otol Neurotol 2001;22:922-927.

8. Selesnick SH, Johnson G. Radiologic surveillance of acoustic neuromas. Am J Otol 1998;19:846-849.

9. Charabi S, Thomsen J, Mantoni M, et al. Acoustic neuroma (vestibular schwannoma): growth and surgical and nonsurgical consequences of the wait-and-see policy. Otolaryngol Head Neck Surg 1995;113:5-14.

10. Olivecrona H. Analysis of results of complete and partial removal of acoustic neuromas. J Neurol Neurosurg Psychiatry 1950;13:271-272.

11. House WF, Luetje CM. Acoustic tumors. Baltimore: University Park Press, 1979.

12. Ohta S, Yokoyama T, Nishizawa S, Uemura K. Regrowth of the residual tumour after acoustic neurinoma surgery. Br J Neuro s u rg 1998;12: 419-422.

13. Aguiar PH, Tatagiba M, Samii M, Dankoweit-Timpe E, Ostertag H. The comparison between the growth fraction of bilateral vestibular schwannomas in neurofibromatosis 2 (NF2) and unilateral vestibular schwannomas using the monoclonal antibody MIB 1. Acta Neurochir (Wien) 1995;134:40-45.

14. Niemczyk K, Vaneecloo FM, Lemaitre L, et al. The growth of acoustic neuromas in volumetric radiologic assessment. Am J Otol 1999;20: 244-248.

15. Charabi S, Engel P, Jacobsen GK, Tos M, Thomsen J. Growth rate of acoustic neuroma expressed by Ki-67 nuclear antigen versus symptom duration. Ann Otol Rhinol Laryngol 1993;102:805-809.

16. Charabi S, Engel P, Charabi B, et al. Growth of vestibular schwannomas: in situ model employing the monoclonal antibody Ki-67 and DNA flow cytometry. Am J Otol 1996;17:301-306.

17. Bedavanija A, Brieger J, Lehr HA, Maurer J, Mann WJ. Association of proliferative activity and size in acoustic neuroma: implications for timing of surgery. J Neurosurg 2003;98:807-811.

18. Fucci MJ, Buchman CA, Brackmann DE, Berliner KI. Acoustic tumor growth: implications for treatment choices. Am J Otol 1999;20:495-499.

19. Hwang SK, Kim DG, Paek SH, et al. Agg ressive vestibular schwannomas with postoperative rapid growth: clinicopathological analysis of 15 cases. Neurosurgery 2002;51:1381-1391. Comment in: Neurosurgery 2003;53:786; author reply 786-787.

20. Chen JM, Houle S, Ang LC, Commins D, Allan K, Nedzelski J, Rowed D. A study of vestibular schwannomas using positron emission tomography and monoclonal antibody Ki-67. Am J Otol 1998;19:840-845. 